\title{
THE LIPIDS OF SERUM IN PREGNANCY ${ }^{1}$
}

\author{
By JOHN P. PETERS, MARTIN HEINEMANN, AND EVELYN B. MAN \\ (From the Department of Internal Medicine, Yale University School of Medicine, \\ New Haven, Conn.)
}

(Submitted for publication December 9, 1950; accepted, February 5, 1951)

It has been repeatedly demonstrated that the concentration of cholesterol in the serum of women increases during pregnancy (1-8). Fatty acids $(1,6,7)$ and phospholipids $(4,6,7)$ also rise somewhat. There is not such general agreement concerning the time relations of the elevations nor the relative changes of the various lipid components. Bloor and Knudson (9) claimed that the increment of cholesterol is composed chiefly of esters; Gardner and Gainsborough (5) found that it consisted entirely of free cholesterol; while, according to Boyd (7), the partition of cholesterol is unaltered. These disagreements can probably be attributed chiefly to the different analytical techniques employed.

In a study of the effect of pregnancy on the precipitable iodine of the serum, lipids were also measured in a number of cases in order to define more precisely the course of the lipids during pregnancy and also to evaluate the significance of the changes of SPI during this process.

\section{MATERIAL AND METHODS}

The patients studied were under the supervision of members of the metabolic division of the Department of Internal Medicine. The majority were delivered in the New Haven Hospital. Blood was taken in the morning. The patients were either fasting or else had a meal containing carbohydrate, but no appreciable amounts of fat or protein (fruit juice, black coffee with sugar, toast without butter, with a little jam or jelly if desired). SPI and serum lipids were measured by methods previously described (10).

\section{RESULTS}

Altogether 145 observations were made on 34 normal persons and 19 observations on five patients with hyperthyroidism under treatment with propylthiouracil and urea. SPI and all lipid fractions (total and free cholesterol, fatty acids and lipid phosphorus) of 31 normal subjects were measured on 114 occasions. In one instance SPI was

1 This investigation was supported (in part) by a research grant from the U. S. Public Health Service. omitted and in a number of others fractionation of lipids was incomplete.

The data from the normal subjects are presented in Table I. Although the interrelationships of the lipid fractions are not entirely unaffected by pregnancy, in general all fractions are altered synchronously and in the same direction. In every case in which repeated observations were made the lipids rise as pregnancy advances. The rise, which is extremely variable in degree, is seldom evident before 12 or more weeks have elapsed. Once it has begun, the lipids appear to increase progressively until term. After delivery they decline gradually, presumably to prepregnant concentration. The word "presumably" is used because, for obvious reasons, actual prepregnancy values are not known. There is reason to suspect that the concentrations observed in early pregnancy do not represent normal nonpregnant values. With a few exceptions, until the lapse of 12 weeks they tend to lie somewhat below the accepted average for a group of normal individuals. In a number of subjects who had more than one observation in the early weeks, cholesterol declined distinctly at this time: MMcM, C46864, MWa. The cholesterol of PG after 10 weeks of pregnancy was $212 \mathrm{mg}$. per cent; but 26 weeks after delivery it was $275 \mathrm{mg}$. per cent. Of course it may not have returned to its normal level in this interval, but it had remained at the same concentration for 15 weeks, definitely lower than it had been in the latter part of pregnancy. The first case, 43773 , is particularly significant because she was studied in two pregnancies. Before the first pregnancy only lipid phosphorus was measured. Values of 9.9 and $10.2 \mathrm{mg}$. per cent were found on two occasions. It had reached practically the same value, $10.5 \mathrm{mg}$. per cent, 32 weeks after delivery. In the early weeks of both pregnancies, however, it fell to between 8 and $9 \mathrm{mg}$. per cent. As evidence that her condition did not differ appreciably on the two occasions, her lipid patterns at 17 and 18 weeks of 
TABLE I

Lipids in normal pregnancy

\begin{tabular}{|c|c|c|c|c|c|c|c|c|c|c|}
\hline \multirow{2}{*}{ Case no. } & \multirow{2}{*}{ Age } & \multirow{2}{*}{ Weeks* } & \multirow{2}{*}{ SPI } & \multicolumn{3}{|c|}{ Cholesterol } & \multirow{2}{*}{$\begin{array}{l}\text { Fatty } \\
\text { acid }\end{array}$} & \multirow{2}{*}{$\underset{P}{\text { Lipid }}$} & \multirow{2}{*}{$\begin{array}{c}\text { Neutral } \\
\text { fat } \\
\text { fatty } \\
\text { acid }\end{array}$} & \multirow{2}{*}{$\frac{\text { Lipid P }-3.7}{\text { Free chol. }}$} \\
\hline & & & & Total & Free & $\frac{\text { Free }}{\text { Total }}$ & & & & \\
\hline 43773 & 29 & $\begin{array}{cc}\text { a } & 5 \\
\text { a } & 2 \\
& 7 \\
& 17 \\
& 30 \\
& 33 \\
& 39 \\
\text { p } & 2 / 7 \\
\text { p } & 12 / 7 \\
\text { p } & 8 \\
\text { p } & 32 \\
& 9 \\
& 13 \\
& 18\end{array}$ & $\begin{array}{l}7.1 \\
5.8 \\
6.2 \\
5.9 \\
4.4 \\
4.1 \\
5.1 \\
4.5 \\
4.9 \\
5.8\end{array}$ & $\begin{array}{l}185 \\
215 \\
211 \\
222 \\
221 \\
217 \\
225 \\
213 \\
160 \\
165 \\
187\end{array}$ & $\begin{array}{l}51 \\
55 \\
64 \\
53 \\
45 \\
69 \\
58 \\
63 \\
42 \\
48 \\
48\end{array}$ & $\begin{array}{l}0.27 \\
0.25 \\
0.30 \\
0.24 \\
0.28 \\
0.32 \\
0.26 \\
0.30 \\
0.26 \\
0.29 \\
0.26\end{array}$ & $\begin{array}{r}m E q / L \\
\\
\\
12.3 \\
14.7 \\
14.8 \\
15.6 \\
14.1 \\
12.2 \\
12.2 \\
13.2 \\
10.7 \\
9.9 \\
12.5\end{array}$ & $\begin{array}{r}m g . \% \\
9.9 \\
10.2 \\
8.3 \\
9.6 \\
11.0 \\
11.3 \\
12.0 \\
11.9 \\
11.1 \\
8.6 \\
10.5 \\
8.8 \\
8.6 \\
9.9\end{array}$ & $\begin{array}{l}3.2 \\
4.1 \\
4.4 \\
4.2 \\
2.6 \\
2.0 \\
2.9 \\
3.2 \\
2.5 \\
1.9 \\
3.2\end{array}$ & $\begin{array}{l}0.116 \\
0.133 \\
0.119 \\
0.157 \\
0.182 \\
0.107 \\
0.102 \\
0.108 \\
0.121 \\
0.102 \\
0.129\end{array}$ \\
\hline C41916 & 25 & $\begin{array}{c}12 \\
15 \\
16 \\
20 \\
23 \\
23 \\
27 \\
31 \\
33 \\
\\
35 \\
\text { p } 2 / 7 \\
\mathrm{p} \quad 6\end{array}$ & $\begin{array}{l}7.9 \\
4.0 \\
6.2 \\
6.6 \\
7.0 \\
7.3 \\
7.0 \\
6.9 \\
8.0 \\
8.9 \\
5.7\end{array}$ & $\begin{array}{l}223 \\
234 \\
243 \\
263 \\
316 \\
342 \\
332 \\
355 \\
333 \\
242\end{array}$ & $\begin{array}{r}48 \\
50 \\
60 \\
70 \\
77 \\
94 \\
100 \\
90 \\
\\
106 \\
75\end{array}$ & $\begin{array}{l}0.22 \\
0.26 \\
0.29 \\
0.29 \\
0.30 \\
0.29 \\
0.27 \\
\\
0.32 \\
0.31\end{array}$ & $\begin{array}{l}11.9 \\
13.6 \\
12.8 \\
17.7 \\
20.7 \\
23.4 \\
23.9 \\
22.5 \\
25.1 \\
27.9 \\
12.5\end{array}$ & $\begin{array}{r}9.3 \\
10.1 \\
10.5 \\
11.6 \\
14.3 \\
14.3 \\
13.3 \\
14.8 \\
14.5 \\
10.3\end{array}$ & $\begin{array}{r}2.4 \\
7.1 \\
9.2 \\
9.6 \\
9.3 \\
8.5 \\
\\
15.8 \\
2.4\end{array}$ & $\begin{array}{l}0.107 \\
0.099 \\
0.102 \\
0.113 \\
0.106 \\
0.107 \\
\\
0.102 \\
0.089\end{array}$ \\
\hline PG & 28 & $\begin{array}{cc} & 10 \\
& 14 \\
& 19 \\
& 23 \\
& 27 \\
& 33 \\
& 37 \\
& 37 \\
\mathrm{p} & 5 / 7 \\
\mathrm{p} & 4 \\
\mathrm{p} & 11 \\
\mathrm{p} & 26\end{array}$ & $\begin{array}{r}8.0 \\
9.5 \\
9.2 \\
10.0 \\
8.5 \\
9.0 \\
8.0 \\
7.9 \\
6.5 \\
5.0 \\
7.0\end{array}$ & $\begin{array}{l}212 \\
273 \\
272 \\
340 \\
355 \\
379 \\
326 \\
349 \\
297 \\
271 \\
275\end{array}$ & $\begin{array}{r}53 \\
75 \\
81 \\
99 \\
83 \\
111 \\
96 \\
84 \\
76 \\
75 \\
76\end{array}$ & $\begin{array}{l}0.25 \\
0.27 \\
0.30 \\
0.29 \\
0.23 \\
0.29 \\
0.29 \\
0.24 \\
0.26 \\
0.28 \\
0.28\end{array}$ & $\begin{array}{l}11.8 \\
17.3 \\
16.9 \\
20.5 \\
20.4 \\
22.3 \\
21.7 \\
16.5 \\
15.3 \\
16.3 \\
14.7\end{array}$ & $\begin{array}{l}10.4 \\
13.0 \\
12.0 \\
14.2 \\
14.3 \\
14.9 \\
14.3 \\
13.2 \\
12.4 \\
12.7\end{array}$ & $\begin{array}{l}1.7 \\
4.7 \\
4.9 \\
6.0 \\
5.0 \\
6.6 \\
7.4 \\
1.8 \\
2.4 \\
3.8\end{array}$ & $\begin{array}{l}0.127 \\
0.124 \\
0.102 \\
0.106 \\
0.127 \\
0.101 \\
0.111 \\
0.113 \\
0.114 \\
0.120\end{array}$ \\
\hline DM & 28 & $\begin{array}{cc} & 9 \\
& 13 \\
22 \\
27 \\
32 \\
\text { p } 3 / 7 \\
\text { p } \quad 6\end{array}$ & $\begin{array}{l}6.5 \\
7.1 \\
7.1 \\
7.3 \\
8.4 \\
7.4 \\
4.7\end{array}$ & $\begin{array}{l}157 \\
156 \\
185 \\
179 \\
178 \\
181 \\
146\end{array}$ & $\begin{array}{l}40 \\
52 \\
49 \\
57\end{array}$ & $\begin{array}{l}0.26 \\
0.28 \\
0.28 \\
0.31\end{array}$ & $\begin{array}{l}10.5 \\
10.6 \\
12.8 \\
14.4 \\
17.9 \\
14.8\end{array}$ & $\begin{array}{l}9.3 \\
8.9 \\
\\
9.4 \\
9.7\end{array}$ & $\begin{array}{l}4.0 \\
5.8 \\
1.6\end{array}$ & $\begin{array}{l}0.107 \\
0.106 \\
0.100\end{array}$ \\
\hline MMcM & 20 & $\begin{array}{cc} & 4 \\
& 8 \\
13 \\
13 \\
20 \\
23 \\
\\
\text { p } & 6 / 7 \\
\text { p } & 3 \\
\text { p } & 8\end{array}$ & $\begin{array}{l}4.9 \\
4.9 \\
6.5 \\
8.9 \\
7.0 \\
5.4 \\
9.4 \\
6.4 \\
5.5\end{array}$ & $\begin{array}{l}172 \\
132 \\
149 \\
156 \\
193 \\
197 \\
233 \\
201 \\
222\end{array}$ & $\begin{array}{l}47 \\
29 \\
37 \\
\\
45 \\
56 \\
64 \\
58\end{array}$ & $\begin{array}{l}0.27 \\
(0.22) \dagger \\
0.25 \\
\\
0.23 \\
0.28 \\
0.27 \\
0.29\end{array}$ & $\begin{array}{r}11.6 \\
8.3 \\
8.3 \\
11.9 \\
16.1 \\
18.0 \\
13.7 \\
11.9 \\
13.1\end{array}$ & $\begin{array}{r}8.8 \\
6.9 \\
7.9 \\
9.1 \\
9.5 \\
9.8 \\
10.1 \\
9.8 \\
8.0\end{array}$ & $\begin{array}{l}3.3 \\
1.6 \\
0.8 \\
\\
6.8 \\
8.6 \\
3.4 \\
1.5\end{array}$ & $\begin{array}{l}0.109 \\
0.114 \\
0.113 \\
\\
0.129 \\
0.109 \\
0.100 \\
0.105\end{array}$ \\
\hline C46864 & 20 & $\begin{array}{c}7 \\
8 \\
9 \\
9 \\
12 \\
16 \\
22 \\
26 \\
31 \\
36 \\
38 \\
40 \ddagger \\
\text { p } 4 / 7 \\
\text { p } 8\end{array}$ & $\begin{array}{r}5.5 \\
6.9 \\
6.3 \\
6.9 \\
8.7 \\
9.8 \\
9.4 \\
8.4 \\
7.3 \\
8.9 \\
8.6 \\
11.2 \\
5.1\end{array}$ & $\begin{array}{l}176 \\
173 \\
160 \\
166 \\
183 \\
219 \\
227 \\
246 \\
270 \\
252 \\
265 \\
277 \\
211\end{array}$ & $\begin{array}{l}45 \\
46 \\
44 \\
51 \\
50 \\
63 \\
65 \\
72 \\
78 \\
71 \\
73 \\
80 \\
52\end{array}$ & $\begin{array}{l}0.26 \\
0.27 \\
0.27 \\
0.31 \\
0.28 \\
0.29 \\
0.29 \\
0.29 \\
0.29 \\
0.28 \\
0.28 \\
0.29 \\
0.25\end{array}$ & $\begin{array}{r}10.5 \\
9.0 \\
10.1 \\
10.3 \\
14.0 \\
16.3 \\
14.8 \\
16.4 \\
18.6 \\
16.4 \\
20.7 \\
18.2 \\
11.6\end{array}$ & $\begin{array}{r}9.0 \\
8.6 \\
7.9 \\
7.7 \\
8.9 \\
11.9 \\
10.7 \\
11.3 \\
11.8 \\
11.9 \\
13.8 \\
14.0 \\
10.4\end{array}$ & $\begin{array}{l}1.9 \\
0.7 \\
2.5 \\
2.8 \\
5.4 \\
5.3 \\
4.4 \\
5.3 \\
6.8 \\
4.8 \\
7.7 \\
4.8 \\
0.2\end{array}$ & $\begin{array}{l}0.118 \\
0.107 \\
0.095 \\
0.075 \\
0.104 \\
0.130 \\
0.108 \\
0.106 \\
0.104 \\
0.115 \\
0.138 \\
0.133 \\
0.129\end{array}$ \\
\hline
\end{tabular}


TABLE I-Continued

\begin{tabular}{|c|c|c|c|c|c|c|c|c|c|c|}
\hline \multirow{2}{*}{ Case no. } & \multirow{2}{*}{ Age } & \multirow{2}{*}{ Weeks* } & \multirow{2}{*}{ SPI } & \multicolumn{3}{|c|}{ Cholesterol } & \multirow{2}{*}{$\begin{array}{l}\text { Fatty } \\
\text { acid }\end{array}$} & \multirow{2}{*}{$\underset{\mathbf{P}}{\operatorname{Lipid}}$} & \multirow{2}{*}{$\begin{array}{c}\text { Neutral } \\
\text { fat } \\
\text { fatty } \\
\text { acid }\end{array}$} & \multirow{2}{*}{$\frac{\text { Lipid P }-3.7}{\text { Free chol. }}$} \\
\hline & & & & Total & Free & $\frac{\text { Free }}{\text { Total }}$ & & & & \\
\hline B85841 & & 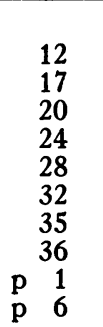 & $\begin{array}{r}\gamma \% \\
6.7 \\
7.8 \\
6.9 \\
7.4 \\
7.1 \\
7.1 \\
9.5 \\
7.7 \\
8.6 \\
4.8\end{array}$ & $\begin{array}{c}m g . \% \\
183 \\
190 \\
212 \\
239 \\
242 \\
242 \\
243 \\
260 \\
244 \\
235\end{array}$ & $\begin{array}{c}\text { mg. \% } \\
54 \\
49 \\
63 \\
70 \\
74 \\
80 \\
74 \\
86 \\
76 \\
65\end{array}$ & $\begin{array}{l}0.29 \\
0.26 \\
0.30 \\
0.29 \\
0.31 \\
0.33 \\
0.30 \\
0.33 \\
0.31 \\
0.28\end{array}$ & $\begin{array}{c}m E q / L \\
12.0 \\
13.2 \\
13.1 \\
15.7 \\
18.2 \\
19.5 \\
19.3 \\
19.8 \\
16.4 \\
15.8\end{array}$ & $\begin{array}{c}m g . \% \\
9.9 \\
10.4 \\
\\
11.8 \\
11.0 \\
12.7 \\
12.5 \\
\\
11.5 \\
10.7\end{array}$ & $\begin{array}{c}m E q / L \\
3.0 \\
3.5 \\
\\
2.7 \\
7.4 \\
7.9 \\
7.6 \\
\\
5.3 \\
5.2\end{array}$ & $\begin{array}{l}0.114 \\
0.136 \\
\\
0.116 \\
0.099 \\
0.112 \\
0.118 \\
\\
0.102 \\
0.108\end{array}$ \\
\hline B78510 $\mid$ & 29 & $\begin{array}{ll} & 16 \\
& 21 \\
& 25 \\
& 31 \\
& 35 \\
& 39 \\
\text { p } & 2 / 7 \\
\text { p } & 11 / 7 \\
\text { p } & 5 \\
\text { p } & 12 \\
\text { p } & 21\end{array}$ & $\begin{array}{l}7.3 \\
6.4 \\
6.3 \\
7.4 \\
6.0 \\
8.8 \\
9.4 \\
7.3 \\
5.0 \\
4.5 \\
5.4\end{array}$ & $\begin{array}{l}244 \\
238 \\
333 \\
350 \\
346 \\
423 \\
385 \\
385 \\
354 \\
292 \\
261\end{array}$ & $\begin{array}{r}66 \\
89 \\
113 \\
144 \\
111 \\
111 \\
112 \\
109 \\
108 \\
80 \\
71\end{array}$ & $\begin{array}{l}0.27 \\
0.37 \\
0.33 \\
0.41 \\
0.32 \\
0.26 \\
0.29 \\
0.28 \\
0.30 \\
0.27 \\
0.27\end{array}$ & $\begin{array}{l}14.6 \\
18.5 \\
21.9 \\
23.0 \\
22.9 \\
24.9 \\
22.4 \\
18.4 \\
18.2 \\
14.8 \\
13.9\end{array}$ & $\begin{array}{l}12.3 \\
12.4 \\
16.0 \\
16.4 \\
14.3 \\
15.0 \\
15.0 \\
14.9 \\
12.6 \\
11.0 \\
10.9\end{array}$ & $\begin{array}{l}2.9 \\
7.4 \\
7.0 \\
8.1 \\
8.5 \\
8.1 \\
6.6 \\
2.5 \\
4.5 \\
2.9 \\
2.7\end{array}$ & $\begin{array}{l}0.130 \\
0.098 \\
0.109 \\
0.088 \\
0.096 \\
0.102 \\
0.101 \\
0.103 \\
0.082 \\
0.091 \\
0.101\end{array}$ \\
\hline C52217 & 32 & 5 & 8.0 & 160 & 49 & 0.31 & 10.0 & 7.6 & 0.1 & 0.080 \\
\hline $\mathrm{HC}$ & & $\begin{array}{l}12 \\
24\end{array}$ & $\begin{array}{l}8.3 \\
7.3\end{array}$ & $\begin{array}{l}168 \\
215\end{array}$ & $\begin{array}{l}45 \\
56\end{array}$ & $\begin{array}{l}0.27 \\
0.26\end{array}$ & $\begin{array}{r}9.3 \\
12.7\end{array}$ & $\begin{array}{r}9.8 \\
11.1\end{array}$ & $\begin{array}{l}0.4 \\
2.2\end{array}$ & $\begin{array}{l}0.135 \\
0.132\end{array}$ \\
\hline MD & 22 & $\begin{array}{c}8 \\
\text { p } 2 / 7\end{array}$ & $\begin{array}{l}8.6 \\
6.7\end{array}$ & $\begin{array}{l}176 \\
264\end{array}$ & 82 & 0.31 & $\begin{array}{l}10.7 \\
18.2\end{array}$ & 13.5 & 6.2 & 0.120 \\
\hline B53121 & 37 & $\begin{array}{c}6 \\
7 \\
328\end{array}$ & $\begin{array}{l}4.6 \\
4.4 \\
8.0\end{array}$ & $\begin{array}{l}205 \\
230 \\
263\end{array}$ & $\begin{array}{l}64 \\
76\end{array}$ & $\begin{array}{l}0.31 \\
0.29\end{array}$ & $\begin{array}{l}12.6 \\
25.7\end{array}$ & 13.1 & 13.2 & 0.124 \\
\hline VD & 34 & 36 & 8.4 & 367 & & & 29.8 & & & \\
\hline CF & 23 & 11 & 6.9 & 225 & 68 & 0.30 & 14.8 & 12.9 & 3.2 & 0.135 \\
\hline C45201 & 22 & 14 & 7.6 & 213 & 60 & 0.28 & 13.5 & 10.6 & 3.4 & 0.115 \\
\hline C13678 & 26 & 3 & 4.0 & 108 & & & 6.4 & & & \\
\hline $\mathrm{AH}$ & & 10 & 7.6 & 197 & 62 & 0.32 & 11.1 & 8.8 & 2.5 & 0.082 \\
\hline C42461 & 23 & $\begin{array}{r}5 \\
7 \\
13 \\
26\end{array}$ & $\begin{array}{l}6.0 \\
6.6 \\
9.0 \\
9.0\end{array}$ & $\begin{array}{l}143 \\
141 \\
141 \\
223\end{array}$ & $\begin{array}{l}39 \\
38 \\
31 \\
58\end{array}$ & $\begin{array}{l}0.27 \\
0.27 \\
0.22 \\
0.26\end{array}$ & $\begin{array}{r}8.1 \\
8.2 \\
7.4 \\
12.8\end{array}$ & $\begin{array}{l}7.8 \\
5.8 \\
6.1 \\
9.2\end{array}$ & $\begin{array}{l}0.9 \\
2.4 \\
1.0 \\
3.2\end{array}$ & $\begin{array}{l}0.106 \\
0.068 \\
0.077 \\
0.095\end{array}$ \\
\hline C15296 & 27 & $\begin{array}{l}34 \\
40\end{array}$ & $\begin{array}{l}4.8 \\
4.8\end{array}$ & $\begin{array}{l}329 \\
266\end{array}$ & 94 & 0.29 & $\begin{array}{l}24.6 \\
31.5\end{array}$ & 15.5 & 9.5 & 0.125 \\
\hline GJ & 28 & 20 & 7.8 & 235 & 78 & 0.33 & 19.4 & 15.4 & 6.4 & 0.150 \\
\hline PJ & & 14 & 6.4 & 161 & 51 & 0.32 & & & & \\
\hline B95180 & 29 & 32 & 6.5 & 252 & 79 & 0.31 & 19.2 & 13.8 & 6.7 & 0.128 \\
\hline $\mathrm{MMcN}$ & & $\begin{array}{l}32 \\
37\end{array}$ & $\begin{array}{l}6.3 \\
6.6\end{array}$ & $\begin{array}{l}292 \\
296\end{array}$ & 79 & 0.27 & 19.9 & 13.8 & 6.4 & 0.128 \\
\hline so & & 8 & 4.3 & 201 & 59 & 0.29 & 12.9 & & & \\
\hline C41546 & 21 & 8 & 6.2 & 201 & 66 & 0.33 & 10.7 & 8.5 & 2.3 & 0.073 \\
\hline B769 & 29 & 12 & 7.0 & 225 & 60 & 0.27 & 11.4 & 9.9 & 1.4 & 0.105 \\
\hline MWa & 28 & $\begin{array}{r}7 \\
11\end{array}$ & $\begin{array}{l}7.6 \\
7.7\end{array}$ & $\begin{array}{l}146 \\
130\end{array}$ & $\begin{array}{l}38 \\
38\end{array}$ & $\begin{array}{l}0.26 \\
0.29\end{array}$ & $\begin{array}{l}9.6 \\
9.5\end{array}$ & $\begin{array}{l}6.3 \\
7.4\end{array}$ & $\begin{array}{l}3.1 \\
2.8\end{array}$ & $\begin{array}{l}0.068 \\
0.074\end{array}$ \\
\hline
\end{tabular}

* Weeks after last menstrual period; a represents weeks before pregnancy; p represents weeks after delivery.

† Parentheses indicate that duplicate values for total or free cholesterol did not check with sufficient accuracy; therefore the ratios are not precise.

† One day before delivery.

One day before premature delivery. For details of case see (12).

II Received $0.02 \mathrm{gm}$. of stilbestrol daily from the end of the 16 th week until delivery. 
the two pregnancies respectively were almost identical. In the second pregnancy the early reduction of lipids involves all fractions proportionally. All these considerations together suggest that the lipids diminish in the early part of pregnancy. If this is the case the data tend to exaggerate the extent of the rise of lipids during pregnancy. This could also be inferred from the fact that the highest values observed in this series seldom exceed the normal limits. In only four subjects were concentrations of cholesterol greater than $300 \mathrm{mg}$. per cent encountered. The rise, nevertheless, appears to be authentic. The general range of lipids in the later part of pregnancy is distinctly above the expectation for a normal group of nonpregnant women. In addition, with the exception of 43773 and MMcM, who was followed for only eight weeks post partum, in those who were followed after delivery, lipids declined. The rises in these two cases were comparatively small. The rate of decline of the lipids is highly variable. There are not sufficient observations to define its course precisely. It apparently does not usually begin until a week or two have elapsed and may not be complete after as long an interval as 12 weeks.

In Table II are a few subjects who received thyroid although they had no evidences of hypothy-

TABLE II

Serum lipids of patients who, although they had no disorder of thyroid function, received thyroid

\begin{tabular}{|c|c|c|c|c|c|c|c|c|c|c|c|c|}
\hline \multirow{2}{*}{ Case no. } & \multirow{2}{*}{ Age } & \multirow{2}{*}{ Weeks* } & \multirow{2}{*}{ SPI } & \multicolumn{3}{|c|}{ Cholesterol } & \multirow{2}{*}{$\begin{array}{l}\text { Fatty } \\
\text { acid }\end{array}$} & \multirow{2}{*}{$\underset{\mathbf{P}}{\text { Lipid }}$} & \multirow{2}{*}{$\begin{array}{c}\text { Neutral } \\
\text { fat } \\
\text { fatty } \\
\text { acid }\end{array}$} & \multirow{2}{*}{$\frac{\text { Lipid } P-3.7}{\text { Free chol. }}$} & \multirow{2}{*}{\multicolumn{2}{|c|}{ Thyroid $†$}} \\
\hline & & & & Total & Free & $\frac{\text { Free }}{\text { Total }}$ & & & & & & \\
\hline $\mathrm{HL}$ & 42 & $\begin{array}{l}26 \\
31 \\
32\end{array}$ & $\begin{array}{l}\gamma \% \\
7.6 \\
5.5 \\
4.8\end{array}$ & $\begin{array}{c}\text { ms. \% } \\
236 \\
235 \\
183\end{array}$ & $\begin{array}{c}\text { mg. } \% \\
65 \\
63 \\
72\end{array}$ & $\begin{array}{c}0.28 \\
0.27 \\
(0.39)\end{array}$ & $\begin{array}{c}m E q / L \\
17.3\end{array}$ & $\begin{array}{c}\text { mg. \% } \\
11.8\end{array}$ & $\begin{array}{c}m E q / L \\
6.1\end{array}$ & 0.125 & $\begin{array}{l}\text { gms. } \\
0.12 \\
0.12\end{array}$ & $\begin{array}{r}w k s . \\
3 \\
4\end{array}$ \\
\hline GM & 31 & $\begin{array}{r}4 \\
12\end{array}$ & $\begin{array}{l}5.2 \\
5.9\end{array}$ & $\begin{array}{l}167 \\
160\end{array}$ & $\begin{array}{l}47 \\
40\end{array}$ & $\begin{array}{l}0.28 \\
0.25\end{array}$ & $\begin{array}{l}10.2 \\
10.4\end{array}$ & 9.3 & 1.9 & 0.140 & 0.12 & 8 \\
\hline HP & 35 & $\begin{array}{l}7 \\
9\end{array}$ & $\begin{array}{l}6.3 \\
9.9\end{array}$ & $\begin{array}{l}160 \\
188\end{array}$ & $\begin{array}{l}83 \\
49\end{array}$ & $\begin{array}{l}0.52 \\
0.26\end{array}$ & $\begin{array}{r}9.5 \\
12.2\end{array}$ & $\begin{array}{r}9.7 \\
10.6\end{array}$ & $\begin{array}{l}1.9 \\
2.5\end{array}$ & $\begin{array}{l}0.072 \\
0.142\end{array}$ & 0.18 & 2 \\
\hline ES & 36 & $\begin{array}{r}7 \\
11 \\
15 \\
27 \\
35\end{array}$ & $\begin{array}{l}3.9 \\
5.8 \\
7.4 \\
5.3 \\
5.4\end{array}$ & $\begin{array}{l}188 \\
187 \\
169 \\
218 \\
255\end{array}$ & $\begin{array}{l}52 \\
49 \\
47 \\
62 \\
71\end{array}$ & $\begin{array}{l}0.28 \\
0.26 \\
0.28 \\
0.28 \\
0.28\end{array}$ & $\begin{array}{l}10.9 \\
10.0 \\
11.0 \\
14.4 \\
16.5\end{array}$ & $\begin{array}{r}10.6 \\
\\
9.3 \\
11.6 \\
11.8\end{array}$ & $\begin{array}{l}1.3 \\
\\
2.4 \\
3.6 \\
4.9\end{array}$ & $\begin{array}{l}0.133 \\
\\
0.119 \\
0.127 \\
0.114\end{array}$ & $\begin{array}{l}0.12 \\
0.24 \\
0.24 \\
0.24\end{array}$ & $\begin{array}{r}4 \\
3 \\
15 \\
23\end{array}$ \\
\hline A63436 & 35 & $\begin{array}{r}5 \\
6 \\
12 \\
16 \\
18 \\
21 \\
238\end{array}$ & $\begin{array}{l}3.5 \\
5.9 \\
8.5 \\
7.9 \\
8.1 \\
8.6 \\
8.4\end{array}$ & $\begin{array}{l}194 \\
172 \\
169 \\
253 \\
221 \\
252 \\
241\end{array}$ & $\begin{array}{l}47 \\
49 \\
35 \\
70 \\
62 \\
73 \\
70\end{array}$ & $\begin{array}{l}0.24 \\
0.28 \\
0.21 \\
0.28 \\
0.28 \\
0.28 \\
0.29\end{array}$ & $\begin{array}{l}13.6 \\
12.2 \\
11.4 \\
17.4 \\
17.2 \\
18.8 \\
14.2\end{array}$ & $\begin{array}{r}10.1 \\
9.6 \\
10.1 \\
12.5 \\
11.8 \\
12.9 \\
11.8\end{array}$ & $\begin{array}{l}3.9 \\
3.4 \\
2.0 \\
5.3 \\
6.3 \\
6.7 \\
3.0\end{array}$ & $\begin{array}{l}0.136 \\
0.120 \\
0.183 \\
0.127 \\
0.130 \\
0.126 \\
0.115\end{array}$ & $\begin{array}{l}0.24 \\
0.30 \\
0.30 \\
0.30 \\
0.42 \\
0.42 \\
0.42\end{array}$ & $\begin{array}{r}1 \\
2 \\
8 \\
12 \\
2 \\
5 \\
7\end{array}$ \\
\hline MW & 22 & 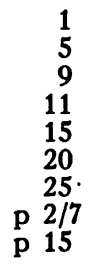 & $\begin{array}{l}4.2 \\
5.5 \\
7.2 \\
7.3 \\
7.8 \\
9.4 \\
7.7 \\
9.7 \\
4.1\end{array}$ & $\begin{array}{l}236 \\
202 \\
165 \\
174 \\
190 \\
228 \\
252 \\
259 \\
233\end{array}$ & $\begin{array}{l}60 \\
56 \\
49 \\
\\
56 \\
\\
73 \\
77 \\
70\end{array}$ & $\begin{array}{l}0.25 \\
0.28 \\
0.30 \\
0.29 \\
0.29 \\
0.30 \\
0.30\end{array}$ & $\begin{array}{r}14.4 \\
11.6 \\
8.8 \\
9.8 \\
12.0 \\
16.4 \\
17.0 \\
18.2 \\
12.3\end{array}$ & $\begin{array}{r}10.0 \\
9.0 \\
8.0 \\
9.1 \\
11.9 \\
10.5\end{array}$ & $\begin{array}{l}3.5 \\
2.6 \\
1.2 \\
\\
3.2 \\
\\
5.5 \\
\\
2.3\end{array}$ & $\begin{array}{l}0.128 \\
0.095 \\
0.088 \\
\\
0.096 \\
\\
0.111 \\
\\
0.090\end{array}$ & $\begin{array}{c}0.18 \\
0.18 \\
0.18 \\
0.18 \\
0.18 \\
0 \\
0\end{array}$ & $\begin{array}{r}3 \\
5 \\
9 \\
14 \\
19 \\
2 / 7 \\
15\end{array}$ \\
\hline TK & 30 & $\begin{array}{l}18 \\
23\end{array}$ & $\begin{array}{l}6.2 \\
6.8\end{array}$ & $\begin{array}{l}279 \\
246\end{array}$ & $\begin{array}{l}77 \\
73\end{array}$ & $\begin{array}{l}0.39 \\
0.34\end{array}$ & $\begin{array}{l}16.3 \\
19.7\end{array}$ & $\begin{array}{l}12.8 \\
13.1\end{array}$ & $\begin{array}{l}3.7 \\
7.3\end{array}$ & $\begin{array}{l}0.118 \\
0.129\end{array}$ & 0.24 & 4 \\
\hline
\end{tabular}

* Weeks after last menstrual period; $p$ represents weeks after delivery.

The number of weeks that the stated dose of thyroid had been taken before the determination.

$\mp$ Parentheses indicate that duplicate values for total cholesterol did not check with sufficient accuracy; therefore the ratios are not precise.

$\$$ On the day of miscarriage. For details of case see (12). 
roidism. The general course of the lipids in these cases is not distinguished from that of the women who did not receive thyroid. This bears out the impression, expressed earlier $(11,12)$ that the pregnant woman tolerates or utilizes thyroid with facility. The lipids may be temporarily depressed after institution of thyroid therapy or an increase of the dose, but rise again shortly. This is evident in ES, A63436 and MW. Observations were not continued long enough in HL and TK to ascertain whether the drops were transitory or not.

Free cholesterol followed the course of total cholesterol quite closely. The ratio, free:total cholesterol, in the serum of normal persons and patients with disorders of the thyroid (10) does not depart from the narrow limits, 0.24 to 0.32 . In 138 observations in this study the ratio exceeded 0.32 only 11 times and 0.33 only seven times. One of these, in HL (Table II), must be discounted because the analysis was not satisfactory. In TK (Table II) the chloresterol ratio was 0.39 after 18 weeks, 0.34 after 23 weeks. No disorder was found to explain these abnormal ratios. The pregnancy proceeded to a normal delivery. In HP (Table II) at the end of seven weeks the ratio was 0.52 , but two weeks later it was only 0.26 . A ratio of 0.34 was found in 98175 (Table III) two days after delivery. Ratios of $0.37,0.33$ and 0.41 were found in B78510 after 21, 25 and 31 weeks, respectively. This subject had normal ratios after 16 and 31 weeks and thereafter on seven different occasions, the last 21 weeks after delivery. This is the woman whose total cholesterol rose highest during pregnancy, reaching $423 \mathrm{mg}$. per cent at the end of 39 weeks. There was, however, no correlation between total cholesterol and the cholesterol ratio. The latter had fallen to 0.26 when the former reached its peak. The subject had had infectious hepatitis three years earlier, but had no demonstrable residua of this disease. She received stilbestrol throughout her pregnancy, being the only subject thus treated. However, the ratio returned to normal while she was still receiving stilbestrol, while the lipids remained high after it was discontinued. The ratio was less than 0.24 in

TABLE III

Serum lipids of patients with disorders of the thyroid

\begin{tabular}{|c|c|c|c|c|c|c|c|c|c|c|c|c|c|c|}
\hline \multirow{2}{*}{ Case no. } & \multirow{2}{*}{ Age } & \multirow{2}{*}{ Weeks* } & \multirow{2}{*}{ SPI } & \multirow{2}{*}{ BEI } & \multicolumn{3}{|c|}{ Cholesterol } & \multirow{2}{*}{$\begin{array}{l}\text { Fatty } \\
\text { acid }\end{array}$} & \multirow{2}{*}{ Lipid } & \multirow{2}{*}{$\begin{array}{c}\text { Neutral } \\
\text { fat } \\
\text { fatty } \\
\text { acid }\end{array}$} & \multirow{2}{*}{$\frac{\text { Lipid P - 3.7 }}{\text { Free chol. }}$} & \multirow{2}{*}{$\begin{array}{l}\text { Lugol's } \\
\text { solution }\end{array}$} & \multirow{2}{*}{\multicolumn{2}{|c|}{ Propylthiouracil }} \\
\hline & & & & & Total & Free & $\frac{\text { Free }}{\text { Total }}$ & & & & & & & \\
\hline C24644 & 34 & $\begin{array}{cc} & 6 \\
& 14 \\
& 20 \\
& 30 \\
\mathrm{p} & 1 / 7 \\
\mathrm{p} & 5\end{array}$ & $\begin{array}{r}\gamma \% \\
9.6 \\
11.3 \\
9.1 \\
8.9 \\
9.1 \\
7.7\end{array}$ & $\begin{array}{l}7.2 \\
7.6 \\
4.7\end{array}$ & $\begin{array}{c}\text { mg. \% } \\
182 \\
253 \\
252 \\
276 \\
\\
243\end{array}$ & $\begin{array}{c}\text { mg. \% } \\
47 \\
74 \\
77 \\
86\end{array}$ & $\begin{array}{l}0.26 \\
0.29 \\
0.31 \\
0.31\end{array}$ & $\begin{array}{c}m E q / L \\
10.6 \\
16.3 \\
16.4 \\
20.2 \\
19.5\end{array}$ & $\begin{array}{r}\text { mg. \% } \\
8.3 \\
11.9 \\
11.9 \\
13.9 \\
13.1\end{array}$ & $\begin{array}{c}m E q / L \\
2.2 \\
4.8 \\
\\
7.2\end{array}$ & $\begin{array}{l}0.098 \\
0.111 \\
0.119\end{array}$ & $\begin{array}{l}g t t s . \\
12 \\
12 \\
12 \\
12 \\
12\end{array}$ & $\begin{array}{r}\text { mg. } \\
100 \\
75 \\
75 \\
75 \\
\\
100\end{array}$ & $\begin{array}{c}\text { woks, } \dagger \\
25 \\
7 \\
13 \\
23 \\
5\end{array}$ \\
\hline 98175 & 32 & $\begin{array}{r}10 \\
14 \\
21 \\
24 \\
26 \\
\text { p } 2 / 7\end{array}$ & $\begin{array}{r}9.4 \\
14.9 \\
8.6 \\
14.9\end{array}$ & $\begin{array}{r}6.9 \\
13.7 \\
6.4 \\
14.0 \\
12.9 \\
16.4\end{array}$ & $\begin{array}{l}257 \\
242 \\
342 \\
278 \\
318 \\
305\end{array}$ & $\begin{array}{r}73 \\
75 \\
94 \\
78 \\
\\
105\end{array}$ & $\begin{array}{l}0.28 \\
0.31 \\
0.28 \\
0.28 \\
\\
0.34\end{array}$ & $\begin{array}{l}16.8 \\
18.0 \\
24.6 \\
25.3 \\
25.2 \\
22.7\end{array}$ & $\begin{array}{l}10.4 \\
11.7 \\
15.3 \\
\\
14.6 \\
14.9\end{array}$ & $\begin{array}{l}6.0 \\
6.9 \\
9.3\end{array}$ & $\begin{array}{l}0.072 \\
0.107 \\
0.099 \\
\\
0.107\end{array}$ & $\begin{array}{l}15 \\
15 \\
15 \\
15 \\
15 \\
15\end{array}$ & $\begin{array}{l}450 \\
450 \\
500 \\
300 \\
300 \\
150\end{array}$ & $\begin{array}{r}6 \\
10 \\
7 \\
3 \\
5 \\
8\end{array}$ \\
\hline A25444 & 26 & $\begin{array}{l}18 \\
23 \\
28\end{array}$ & $\begin{array}{r}11.2 \\
8.6\end{array}$ & $\begin{array}{r}6.7 \\
6.1 \\
11.8\end{array}$ & $\begin{array}{l}200 \\
349 \\
159\end{array}$ & $\begin{array}{l}59 \\
73 \\
49\end{array}$ & $\begin{array}{l}0.29 \\
0.21 \\
0.31\end{array}$ & $\begin{array}{l}16.2 \\
19.1 \\
12.5\end{array}$ & $\begin{array}{l}13.4 \\
14.0\end{array}$ & $\begin{array}{l}4.8 \\
3.8\end{array}$ & $\begin{array}{l}0.164 \\
0.141\end{array}$ & $\begin{array}{l}7 \\
7 \\
7\end{array}$ & $\begin{array}{l}300 \\
300 \\
150\end{array}$ & $\begin{array}{r}8 \\
13 \\
5\end{array}$ \\
\hline B94697 & 36 & $\begin{array}{cc} & 37 \\
& 40 \\
\text { p } & 2 / 7 \\
\text { p } & 2\end{array}$ & $\begin{array}{r}8.7 \\
11.3 \\
10.5 \\
11.1\end{array}$ & 6.8 & $\begin{array}{l}186 \\
189 \\
173 \\
270\end{array}$ & $\begin{array}{l}54 \\
59 \\
72\end{array}$ & $\begin{array}{l}0.29 \\
0.31 \\
0.27\end{array}$ & $\begin{array}{l}19.9 \\
20.2 \\
17.4 \\
21.1\end{array}$ & $\begin{array}{l}11.5 \\
10.9 \\
11.6\end{array}$ & $\begin{array}{r}10.8 \\
10.5 \\
9.2\end{array}$ & $\begin{array}{l}0.144 \\
0.122 \\
0.110\end{array}$ & $\begin{array}{l}10 \\
10 \\
10 \\
10\end{array}$ & $\begin{array}{l}50 \ddagger \\
25 \ddagger \\
25 \ddagger \\
25 \ddagger\end{array}$ & $\begin{array}{r}16 \\
3 \\
3 \\
5\end{array}$ \\
\hline C19922 & 36 & 19 & 9.2 & & 179 & 48 & 0.27 & 15.2 & & & & 14 & 300 & 14 \\
\hline
\end{tabular}

* Weeks after last menstrual period; $p$ represents weeks after delivery.

$\uparrow$ Number of weeks patients had been taking propylthiouracil.

$\ddagger$ Thiourea instead of propylthiouracil. 
three subjects. One of these can be attributed to analytical error. No cause for abnormally low ratios has been discovered. In no case could variations of the ratio, within or outside of the normal range, be related to the stage of pregnancy or to other characteristics of the lipids. The deviations from the normal range must, therefore, be attributed to adventitious disorders, the nature of which was not discovered.

In normal persons the relationship of phospholipid to cholesterol is most precisely defined by the equation, $\frac{\mathrm{mg} \text {. per cent of lipid } \mathrm{P}-3.7}{\mathrm{mg} \text {. per cent of free cholesterol }}=0.106$ (13). In the present study the average ratio was 0.113 , varying from 0.067 to 0.182 . No significance can be attached to the slightly elevated average ratio or the variability of the ratio. These features could not be correlated with the course of pregnancy, other lipid fractions or the cholesterol ratio.

The most distinctive feature of the lipid pattern is an increase of the concentration of neutral fat as pregnancy progresses. Although this is usually moderate and varies from case to case, it is unmistakable and consistent in all subjects and therefore significant. Neutral fat and cholesterol appear to be roughly correlated; but the correlation probably derives only from the fact that both rise as pregnancy progresses. Neither in individual cases nor in the series as a whole do the two functions vary proportionally. Neutral fat appears to fall earlier than cholesterol does after delivery.

Both SPI and cholesterol rise as pregnancy advances. There is no reason except this association to connect the two phenomena. In most other circumstances cholesterol tends to diminish as SPI rises.

In Table III are five pregnant women with hyperthyroidism who were treated with this drug and Lugol's solution of iodine. Although the lipids are more variable in these patients than they are in normal women they have the same tendency to rise as pregnancy advances and to decline after delivery. SPI in these cases does not mirror precisely the degree of thyroid activity for reasons pointed out elsewhere. SPI may rise further in hyperthyroid than in normal persons during pregnancy (12). In addition administration of iodine causes spurious increases of SPI (14). Butanol-extractable iodine (BEI), figures for which are given in sev- eral instances, is a better measure of thyroid activity in these cases. In A25444 and 98175 the lipids fell when signs of frank hyperthyroidism appeared with BEI's above 10 gamma per cent. In the latter case, they rose again in spite of the continued hyperthyroidism.

\section{DISCUSSION}

There is little to add to this description. The causes of these variations in the concentrations and interrelationships during pregnancy will remain conjectural until the general metabolism of pregnancy has been further elucidated. The early decline of lipids, if it is verified, may be related to vomiting of pregnancy, but this was not conspicuous in 43773 (Table I), whose lipids fell with the onset of both pregnancies. It has been shown that certain estrogenic materials increase the lipids of the serum and livers of rats and guinea pigs (15-17). Increases of neutral fat are reported to occur either after meals or when fat is being mobilized rapidly from the depots to the liver for formation of ketone bodies. It is intriguing to speculate about the possible connection of this phenomenon in pregnancy with the recognized tendency of the pregnant woman to spend carbohydrate prodigally and her unusual susceptibility to ketosis. Variability of the decline of lipids after delivery may be connected with lactation; but no such connection could be discerned in this small series of subjects. If the hyperlipemia were related to the great discharge of estrogenic materials that characterizes pregnancy such a delay should not be expected.

The association of hyperlipemia with high SPI might be interpreted as evidence of relative hypothyroidism. It is conceivable that the demands of the tissues for the thyroid hormone and utilization of the hormone are augmented during pregnancy and that the increased discharge of the hormone manifested in the elevated SPI is not sufficient to meet these demands. This is an unlikely explanation for two reasons. When the hyperthyroid patient, 98175 , lapsed into overt hyperthyroidism, although the lipids fell they still remained far higher than they would have been in a hyperthyroid nonpregnant woman. Moreover in the hyperlipemia of hypothyroidism neutral fat does not rise (10). 


\section{SUMMARY AND CONCLUSIONS}

The lipids and precipitable iodine (SPI) of the serum of 34 normal women and five women with medically treated hyperthyroidism have been measured at intervals during pregnancy and after delivery. There is evidence to suggest that the lipids may decline slightly in the early weeks of pregnacy. After about the 12th week, however, they rise progressively until delivery, thereafter declining at a variable rate. In this rise total and free cholesterol and phospholipids participate proportionally, maintaining their normal relations to one another. Neutral fat, however, rises proportionally far more than the other lipid fractions and declines more rapidly than the others after delivery.

\section{REFERENCES}

1. Hermann, E., and Neumann, J., Uber den Lipoidgehalt des Blutes normaler und schwangerer Frauen sowie neugeborener Kinder. Biochem. Ztschr., 1912, $43,47$.

2. Stepp, W., Ueber den Cholesteringehalt des Blutserums bei Krankheiten. München. med. Wchnschr., 1918, 65, 781.

3. Pribram, E. E., Zur Frage des Cholesterinstoffwechsels während der Schwangerschraft und im Wochenbett. Arch. f. Gynaek., 1923, 119, 57.

4. Oser, B. L., and Karr, W. G., The lipoid partition in blood, in health and in disease. Arch. Int. Med., 1925, 36, 507.

5. Gardner, J. A., and Gainsborough, H., The cholesterol metabolism during pregnancy. Lancet, 1929, 1, 603.

6. Fahrig, C., and Wacker, L., Vergleichende Untersuchungen über der Lipoidkomplex des Blusterums bei essentieller Hypertension, Muskelarbeit, Hunger, Schwangerschaft und Nahrungsaufnahme. Klin. Wchnschr., 1932, 11, 886.

7. Boyd, E. M., The lipemia of pregnancy. J. Clin. Invest., 1934, 13, 347.

8. Boyd, E. M., Blood lipids in the puerperium. Am. J. Obst. \& Gynec., 1935, 29, 797.

9. Bloor, W. R., and Knudson, A., The separate determination of cholesterol and cholesterol esters in small amounts of blood. J. Biol. Chem., 1916, 27, 107.

10. Peters, J. P., and Man, E. B., The significance of serum cholesterol in thyroid disease. J. Clin. Invest., 1950, 29, 1.

11. Man, E. B., Heinemann, M., Johnson, C. E., Leary, D. C., and Peters, J. P., The precipitable iodine of serum in normal pregnancy and its relation to abortions. J. Clin. Invest., 1951, 30, 137.

12. Engstrom, W. W., Kydd, D. M., Peters, J. P., and Man, E. B., The precipitable iodine of serum in pregnancy complicated by disorders of the thyroid. J. Clin. Invest., 1951, 30, 151.

13. Albrink, M. J., Man, E. B., and Peters, J. P., Serum lipids in infectious hepatitis and obstructive jaundice. J. Clin. Invest., 1950, 29, 781.

14. Man, E. B., Kydd, D. M., and Peters, J. P., Butanolextractable iodine of serum. Submitted for publication.

15. Bogdanovitch, S. B., and Man, E. B., The effects of castration, theelin, testosterone and antuitrin-S on the lipoids of blood, liver and muscle of guinea pigs. Am. J. Physiol., 1938, 122, 73.

16. Loeb, H. G., Effect of estradiol benzoate on serum lipid of rats consuming a high fat diet. Proc. Soc. Exper. Biol. \& Med., 1942, 49, 340.

17. Loeb, H. G., Influence of estradiol benzoate on fat storage. Proc. Soc. Exper. Biol. \& Med., 1942, 51, 330. 